\title{
Spin Relaxation Measurements Using First-Harmonic Out-of-Phase Absorption EPR Signals
}

\author{
V. A. Livshits, ${ }^{*}$ T. Páli, $\dagger^{, 1}$ and D. Marsh $\dagger^{, 2}$ \\ *Centre of Photochemistry, Russian Academy of Sciences, 117427, Moscow, Russian Federation; and †Max-Planck-Institut \\ für biophysikalische Chemie, Abteilung Spektroskopie, D-37070 Göttingen, Germany
}

Received August 18, 1997; revised April 7, 1998

The dependence on spin-lattice $\left(T_{1}\right)$ relaxation of the firstharmonic absorption EPR signal $\left(V_{1}^{\prime}\right)$ detected in phase quadrature with the Zeeman modulation has been investigated both theoretically and experimentally for nitroxide spin labels. Spectral simulations were performed by iterative solution of the Bloch equations that contained explicitly both the modulation and microwave magnetic fields (T. Páli, V. A. Livshits, and D. Marsh, 1996, J. Magn. Reson. B 113, 151-159). It was found that, of the various non-linear E PR displays, the first-harmonic out-of-phase $V_{1}^{\prime}$-signal, recorded under conditions of partial saturation of the microwave absorption, is particularly favorable for determining spin- lattice relaxation enhancements because of its superior signal intensity and relative insensitivity to spin-spin $\left(T_{2}\right)$ relaxation. By varying the Zeeman modulation frequency it is also possible to tune the optimum sensitivity of the $V_{1}^{\prime}$-signal to different ranges of the $T_{1}$-relaxation time. A Zeeman modulation frequency of $25 \mathrm{kHz}$ appears to be particularly suited to spin label applications. Calibrations are given for the dependence on $T_{1}$-relaxation time of both the amplitude and the second integral of the $V_{1}^{\prime}$-signal recorded under standard conditions. Experiments on different spin labels in solution and in membranes demonstrate the practical usable sensitivity of the $V_{1}^{\prime}$-signal, even at modulation frequencies of $25 \mathrm{kHz}$, and these are used to investigate the dependence on microwave field intensity, in comparison with theoretical predictions. The practicable sensitivity to spin-lattice relaxation enhancements is demonstrated experimentally for a spin-labeled membrane system in the presence of paramagnetic ions. The first-harmonic out-of-phase $V_{1}^{\prime}$-signal appears to be the non-linear CW EPR method of choice for determining $T_{1}$-relaxation enhancements in spin-labeled systems. $\odot 1998$ Academic Press

Key Words: non-linear E PR ; spin-lattice relaxation; spin labels; out-of-phase EPR.

\section{INTRODUCTION}

The response of a spin system to modulation of the Zeeman interaction is known to be phase-shifted relative to the harmonically modulated applied field because of the finite spin relaxation times of the system (1). This shift in phase is usually treated in

\footnotetext{
${ }^{1}$ Permanent address: Institute of Biophysics, Biological Research Center, H-6701 Szeged, Hungary.

${ }^{2}$ To whom correspondence should be addressed.
}

terms of two components of the EPR signal: those in-phase and those out-of-phase with respect to the static field modulation. It is the latter component that records specifically the phase retardation effects, and therefore it must be more sensitive to spin relaxation times than is the conventional in-phase absorption component.

The absorption EPR spectra of spin labels detected at the second harmonic, in phase quadrature with respect to the modulation field (i.e., the saturation transfer EPR spectra, $V_{2}^{\prime}$ ) have been used recently to determine spin-lattice relaxation enhancements induced by Heisenberg spin exchange or by dipole-dipole interactions with fast relaxing paramagnetic species $(2,3)$. These new applications of ST EPR spectroscopy, as well as conventional $\mathrm{CW}$ progressive saturation measurements, offer considerable promise for structural studies of biological membranes because they allow determination of the location of site-specifically labeled groups in membrane proteins and in phospholipids (4-7). Because the intrinsic spin-lattice relaxation times $\left(T_{1}\right)$ of nitroxyl spin labels are usually 1-2 orders of magnitude larger than the spin-spin relaxation times $T_{2}(8,9)$, the former are of primary importance for such studies. The intensities of the $V_{2}^{\prime}$-spectra, although sensitive to $T_{1}$, are also very sensitive to molecular rotation (10) - as is exploited in the usual application of ST EPR spectroscopy to studying slow rotational diffusion - and additionally are rather sensitive to $T_{2}$-relaxation (11). The saturation parameters from progressive saturation EPR experiments, on the other hand, are less sensitive to $T_{1}$ and also are dependent on $T_{2}$ directly $(12,13)$.

Partly for reasons of sensitivity and also of availability and ease of use, most EPR measurements on spin-labeled biological systems are routinely made with conventional CW equipment. Therefore it is particularly useful and relevant to explore possible new non-linear CW EPR approaches for determining the effective spin relaxation times. The aim of the present work is to investigate the practical utility of measuring spin-lattice relaxation enhancements of spin-labeled biomolecules by using the out-of-phase first-harmonic EPR absorption signals (i.e., $\left.V_{1}^{\prime}\right)$. It is shown here that the amplitudes or double integrals of the $V_{1}^{\prime}$-signal, normalized to the corresponding quantities for the in-phase signals, are more sensitive to $T_{1}$ and are less dependent on $T_{2}$, as compared with the conventional in-phase 
first-harmonic absorption $V_{1}$-spectra. The application of this approach to nitroxide spin labels in solution and in lipid membranes is demonstrated.

It should be emphasized that the spin-lattice relaxation times derived from $\mathrm{CW}$ saturation methods are effective values, because they contain contributions from nuclear relaxation. In general, they are smaller than the intrinsic values determined by timedomain methods such as saturation recovery $(8,9)$. This point has been dealt with in some detail in a previous publication (14). The application of CW methods with spin labels is, however, restricted mostly to determining spin-lattice relaxation enhancements, rather than absolute values of $T_{1}$. An example of this that is directly related to structural determinations is given here. The principal advantage of the present method, relative to other $\mathrm{CW}$ saturation methods, is that it is insensitive to $T_{2}$. This is most important when comparing spin-lattice relaxation enhancements from systems with different linewidths.

\section{THEORY}

For simplicity, we consider first a single-line EPR spectrum in the absence of molecular motion. The Bloch equations that include the microwave $H_{1}$-field and the Zeeman modulation field are solved as described in Ref. (11), by following the method of Ref. (15). A Fourier expansion is made for the magnetization components $(u, \mathrm{v}, w)$ in the rotating frame, in terms of the harmonics, $n$, of the Zeeman modulation frequency, $\omega_{m}$. The resulting Fourier coefficients $\left(u_{n}, \mathrm{v}_{n}, w_{n}\right)$ are then expanded in a power series of the Zeeman modulation amplitude, $H_{m}$. For the $n$th harmonic, the first non-vanishing coefficients $\left(u_{n, n}, \mathrm{v}_{n, n}, w_{n, n}\right)$ depend on the $n$th power of the field modulation. The lineshapes of the in-phase and out-ofphase absorption signals, $V_{n, n}$ and $V_{n, n}^{\prime}$, are given by the real and imaginary parts, respectively, of the Fourier coefficient $\mathrm{V}_{n, n}$. Expressions for the absorption lineshapes detected at the first-harmonic, both in-phase and out-of-phase with respect to the field modulation, and under conditions of partial microwave power saturation, are obtained by iterative solution of the Bloch equations (see Refs. $(11,15))$

$$
\begin{aligned}
& V_{1,1}=-V_{0,0} D \frac{2\left(D^{2}+A\right)-B \omega^{\prime 2}}{\left(D^{2}+A\right)^{2}+B^{2} \omega^{\prime 2}} \\
& V_{1,1}^{\prime}=\omega^{\prime} \cdot V_{0,0} D \frac{D^{2}+A+2 B}{\left(D^{2}+A\right)^{2}+B^{2} \omega^{\prime 2}},
\end{aligned}
$$

where

$$
\begin{aligned}
& A=1-\omega^{\prime 2}+h^{2} \frac{a+\omega^{\prime 2}}{a^{2}+\omega^{\prime 2}} \\
& B=-2+h^{2} \frac{1-a}{a^{2}+\omega^{\prime 2}}
\end{aligned}
$$

and $a=T_{2} / T_{1}$. In Eqs. [1]-[3], angular frequencies are expressed in terms of the transverse relaxation time, $T_{2}$, i.e.,

$$
\begin{aligned}
\omega^{\prime} & =\omega_{m} T_{2} \\
h & =\gamma_{e} H_{1} T_{2} \\
D & =\left(\gamma_{e} H-\omega\right) T_{2},
\end{aligned}
$$

where $\gamma_{e}$ is the electron gyromagnetic ratio, $\omega$ is the angular microwave frequency, and $H$ is the static magnetic field strength. The zeroth harmonic (in-phase) solution is given by the normal slow-passage absorption lineshape (11), i.e.,

$$
V_{0,0}=\frac{h}{1+D^{2}+s}
$$

where $s=h^{2} / a$ is the slow-passage saturation parameter. Here, the zeroth harmonic absorption is defined as having positive polarity.

In addition to the spectral amplitudes, the second integral of the out-of-phase $V_{1,1}^{\prime}$-signal and the out-of-phase/in-phase integral ratio

$$
\rho_{1}^{\prime}=\frac{\iint V_{1,1}^{\prime} d^{2} D}{\iint V_{1,1} d^{2} D}
$$

are also of interest, because the double integrals of the EPR spectra under saturation are known not to depend on the inhomogeneous broadening (13). In order to investigate the functional dependences on spin relaxation times, as well as on the experimental parameters $H_{1}$ and $\omega_{m}$, analytical expressions are derived for the integrals by using the reasonable approximation that $A^{2} \gg$ $B^{2} \omega^{\prime 2}$. For nitroxides in liquid solutions, the usual ranges for $T_{1}$ and $T_{2}$ are $5 \times 10^{-7}-10^{-5}$ and $10^{-8}-10^{-7} \mathrm{~s}$, respectively $(8,9)$. In these ranges, the following inequalities hold for $\omega_{m} / 2 \pi \leq 100$ $\mathrm{kHz}: a \ll 1, \omega^{\prime} \ll 1$, and $\omega^{\prime 2} / a=\omega_{m}^{2} T_{1} T_{2} \ll 1$, which lead to approximations for $A$ and $B$ that are given by

$$
\begin{aligned}
& A \approx 1+\frac{h^{2} a}{a^{2}+\omega^{\prime 2}} \\
& B \approx-2+\frac{h^{2}}{a^{2}+\omega^{\prime 2}} .
\end{aligned}
$$

The condition $A^{2} \gg B^{2} \omega^{\prime 2}$ is then equivalent to the inequality

$$
\frac{1}{\omega_{m} T_{1}}(1+1 / s)+\frac{\omega_{m} T_{1}}{s}>1,
$$

which always holds for $\omega_{m} T_{1}<1$, and also for $\omega_{m} T_{1}>1$ in the case of low saturation (i.e., $s \leq 1$ ).

Under these approximations, which allow one to neglect the 
second terms in the denominators of Eqs. [1] and [2] and the second term in the numerator of Eq. [1], the expressions for the second integrals of the in-phase and out-of-phase first-harmonic absorption, and for the out-of-phase/in-phase integral ratio $\rho_{1}^{\prime}$ are

$$
\begin{aligned}
\iint V_{1,1} d^{2} D & =\frac{2 \pi h}{\sqrt{A}+\sqrt{1+s}} \\
\iint V_{1,1}^{\prime} d^{2} D & =\frac{-\omega^{\prime} \pi h}{\sqrt{A}+\sqrt{1+s}}\left(1+\frac{B}{A+\sqrt{A(1+s)}}\right) \\
\rho_{1}^{\prime} & =-\omega^{\prime} / 2\left(1+\frac{B}{A+\sqrt{A(1+s)}}\right)
\end{aligned}
$$

where the negative sign in Eq. [11] indicates that the out-of-phase and in-phase signals have opposite polarities. The analytical expressions, given by Eqs. [10] and [11], contain the explicit dependence of the out-of-phase spectral integral and its normalized value, $\rho_{1}^{\prime}$, on the relaxation times $T_{1}$ and $T_{2}$, and also on $H_{1}$ and $H_{m}$.

The microwave saturation of the amplitudes of an EPR spectrum is known to depend on the degree of inhomogeneous broadening. Nevertheless, it is useful to analyze the ratio of amplitudes for the out-of-phase and in-phase first-harmonic absorption EPR signals as an alternative (or supplementary) parameter for the $T_{1}$ measurements, because one may expect that the effects of inhomogeneous broadening are partially compensated in the amplitude ratio (see later). The potential advantage of using this amplitude parameter is the ability to deduce relaxation properties for different spin-label species which give overlapping spectra, or from EPR spectra with an underlying baseline that disturbs measurements of the double integral.

The ratio of the $V_{1^{-}}^{\prime}$ to $V_{1}$-amplitudes at the same field position, $D$, is given from Eqs. [1] and [2] by

$$
\rho_{1}^{\prime}(D)=\frac{V_{1}^{\prime}(D)}{V_{1}(D)}=-\omega^{\prime} \frac{D^{2}+A+2 B}{2\left(D^{2}+A\right)-B \omega^{\prime 2}} .
$$

For the less stringent condition $A \gg B \omega^{\prime 2}$, together with the approximations given by Eqs. [7], the expression for the amplitude ratio becomes

$$
\begin{aligned}
\rho_{1}^{\prime}(\Delta \omega)= & -\frac{\omega_{m} T_{2}}{2}-\frac{\omega_{m} T_{1}}{1+\omega_{m}^{2} T_{1}^{2}} \\
& \times \frac{\gamma_{e}^{2} H_{1}^{2} T_{1} T_{2}}{1+(\Delta \omega)^{2} T_{2}^{2}+\gamma_{e}^{2} H_{1}^{2} T_{1} T_{2} /\left(1+\omega_{m}^{2} T_{1}^{2}\right)},
\end{aligned}
$$

where $\Delta \omega=\gamma_{e}\left(H-H_{o}\right)$ is the distance of the chosen field position, $H$, from the center resonance field, $H_{o}$. It will be noted that the peak-to-peak $V_{1}$ - and $V_{1}^{\prime}$-amplitudes, for motionally narrowed first-harmonic spectra, correspond to different field positions (see Results). Besides the approximate analytical expressions Eqs. [11] and [13] for $\rho_{1}^{\prime}$ and $\rho_{1}^{\prime}(\Delta \omega)$, the exact integral and amplitude ratios were obtained directly from Eqs. [1] and [2], by using numerical integration in the former case. The latter exact results are used for establishing all calibration parameters and for deriving effective spin-lattice relaxation times from experimental data. The approximate analytical expressions are used for illustrative purposes in discussing the sensitivity to relaxation times and $H_{1}$-field intensity.

\section{MATERIALS AND METHODS}

The spin label TEMPONE (4-oxo-2,2,6,6-tetramethylpiperidine- $N$-oxyl) was obtained from Eastman Kodak (Rochester, NY). Spin-labeled phosphatidylcholines, n-PCSL (1-acyl-2-[n(4,4-dimethyloxazolidine- $N$-oxyl)stearoyl]-sn-glycero-3-phosphocholine), were synthesized according to Ref. (16). Synthetic phosphatidylcholines, DMPC (1,2-dimyristoyl-sn-glycero-3phosphocholine) and DPPC (1,2-dipalmitoyl-sn-glycero-3phosphocholine) were from Avanti Polar Lipids (Alabaster, AL).

TEMPONE was used at a concentration of $0.3 \mathrm{~m} M$ in argon-saturated glycerol/water solutions. Spin-labeled phosphatidylcholine, 5-PCSL, was incorporated in bilayer membranes of DMPC or DPPC at a relative concentration of 0.5 mol\%, and correspondingly 16-PCSL was incorporated at 0.7 mol\% (see, e.g., Ref. (11)). All membrane dispersions were saturated with argon. Aliquots of the samples were loaded into $50 \mu \mathrm{l}, 0.7 \mathrm{~mm}$ i.d., glass capillaries and flushed with argon. Sample sizes were trimmed to $5 \mathrm{~mm}$ length to avoid inhomogeneities in the $H_{1}$ and $H_{m}$ fields (17).

EPR spectra were recorded at a microwave frequency of $9 \mathrm{GHz}$ on a Varian Century Line spectrometer equipped with nitrogen gas flow temperature regulation. Sample capillaries were positioned along the symmetry axis of the standard $4 \mathrm{~mm}$ quartz EPR sample tube that contained light silicone oil for thermal stability. Temperature was measured with a fine-wire thermocouple located within the capillary in contact with the sample. Samples were centered in the $\mathrm{TE}_{102}$ rectangular microwave cavity and all spectra were recorded under critical coupling conditions. The root-mean-square microwave magnetic field $\left\langle H_{1}^{2}\right\rangle^{1 / 2}$ at the sample was measured as described in Ref. (17), and corrections were made for the cavity Q as described in the same reference. The in-phase and out-of-phase EPR spectra were recorded in the first-harmonic absorption mode at modulation frequencies of 100 and $25 \mathrm{kHz}$. The modulation field measured at the sample was $0.32 \mathrm{G} \mathrm{p}-\mathrm{p}$ (17). ST EPR spectra, where appropriate, were recorded under standard conditions, as described in Ref. (11). Progressive saturation experiments were performed using the double-integrated intensity of the in-phase $V_{1}$-spectra, essentially as described in Ref. (13).

Correct recording of the $V_{1}^{\prime}$-signals, especially at low $H_{1}$ values, depends crucially on accurate setting of the modulation phase, which in the standard protocols is performed at subsaturating microwave field intensities. The self-null method that is normally used is not always appropriate because the $V_{1}^{\prime}$-signal 

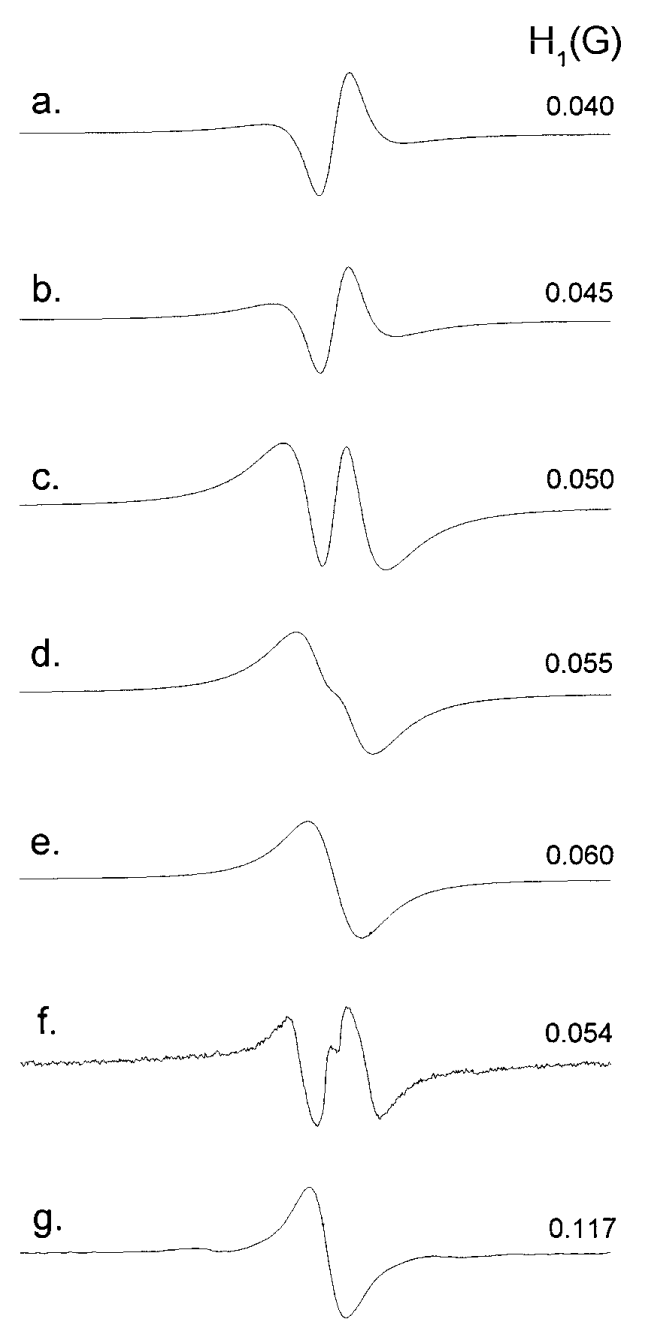

FIG. 1. Dependence of the lineshape of the $90^{\circ}$-out-of-phase $V_{1}^{\prime}$-signal on the $H_{1}$-microwave field for a single EPR line. (a)-(e) Spectra calculated without approximation from Eq. [2] with $T_{1}=2.2 \mu \mathrm{s}, \Delta H=1 / \gamma_{e} T_{2}=1 \mathrm{G}$ and $\omega_{m} / 2 \pi=$ $10^{5} \mathrm{~s}^{-1}$. (f), (g) Experimental $V_{1}^{\prime}$-spectra of the central ${ }^{14} \mathrm{~N}$ hyperfine line of TEMPONE in $90 \%$ glycerol at $20^{\circ} \mathrm{C}$, with $\omega_{m} / 2 \pi=10^{5} \mathrm{~s}^{-1}$. Spectra are normalized to the same height for comparison. Displayed scan width $=15 \mathrm{G}$.

in phase quadrature is not zero, even in the absence of saturation (see Eq. [2] and Fig. 1). The phase therefore was set according to the following considerations of the spectral amplitudes and lineshapes about the region of the quadrature phase setting, for the sample to be measured. The first-harmonic voltage corresponding to the EPR absorption is the real part of the expression

$$
V=\left(V_{1}+i V_{1}^{\prime}\right) e^{-i \omega_{m} t}
$$

i.e.,

$$
\operatorname{Re}\{V\}=V_{1} \cos \omega_{m} t+V_{1}^{\prime} \sin \omega_{m} t
$$

If the phase of the lock-in-amplifier is shifted by an amount
$\Delta \phi$, relative to the phase of the applied modulation field, the observed signal is

$$
\begin{aligned}
S & =\left(\omega_{m} / 2 \pi\right) \int_{0}^{2 \pi / \omega_{m}} \operatorname{Re}\{V\} \cdot \cos \left(\omega_{m} t+\Delta \phi\right) d t \\
& =\frac{1}{2}\left(V_{1} \cos \Delta \phi-V_{1}^{\prime} \sin \Delta \phi\right) .
\end{aligned}
$$

When the phase angle is changed by a small amount, $\chi$, on either side of the quadrature phase setting, $\Delta \phi=\pi / 2 \pm \chi$, where $|\chi| \ll \pi / 2$, the resulting signal is given by $S \cong-\frac{1}{2}\left(V_{1} \chi\right.$ $\left.+V_{1}^{\prime}\right)$; i.e., $S$ is approximately linearly dependent on $\chi$. In the absence of saturation, the $V_{1}^{\prime}$-lineshape resembles that of the $V_{1}$-signal, except in the wings (see Fig. 1a), and the $V_{1}^{\prime} / V_{1}$ amplitude ratio is given approximately by $\omega_{m} T_{2} / 2$. The linear dependence of the signal intensity $S(\chi)$ on the phase increment $\chi$ is distorted, however, in the vicinity of $\Delta \phi=\pi / 2$, because the p-p linewidths of the $V_{1^{-}}$and $V_{1}^{\prime}$-signals differ. The $90^{\circ}$ phase setting was therefore estimated from the linear intercept of $S(\chi)$ with the $\Delta \phi$-axis by using the data points that lie outside the central "distortion" region. The error in this procedure is $\delta \chi \approx V_{1}^{\prime} / V_{1}$, which can be appreciable for sharp lines. Moreover, the condition for no saturation $(B \approx-2$, i.e., $\left(\gamma_{e} H_{1} T_{1}\right)^{2} \ll 1$; see Eq. [3]) is more difficult to achieve for the $V_{1}^{\prime}$-signal than for the in-phase $V_{1}$-signal (viz. $s \ll 1$ ). For more accurate phase settings and also to check for possible phase drift, a non-saturable reference sample was used, because for this the null method is quite adequate at all accessible values of $H_{1}$. The reference sample was a small monocrystal of $\mathrm{CuSO}_{4}$. $5 \mathrm{H}_{2} \mathrm{O}$, which has an anisotropic exchange-narrowed EPR line. This was attached at the side of the sample, in an orientation at which the resonance field lies outside the range of nitroxyl spin label spectra. A phase setting precision of $<0.1^{\circ}$ could be achieved by this latter method.

\section{RESULTS AND DISCUSSION}

\section{Out-of-Phase $V_{1}^{\prime}$-Lineshape}

As can be seen from Eq. [2], the lineshape of the nonlinear $V_{1}^{\prime}$-signal depends strongly on the value of the parameter $B$. At sufficiently low values of $H_{1}$, for which $h^{2}(1-$ a) $/\left(a^{2}+\omega^{2}\right)<2$ (cf. Eq. [3]), the out-of-phase $V_{1}^{\prime}$-lineshape has the form shown by the exact simulations in Figs. 1a-1d. For the reverse condition, which holds already for rather moderate degrees of saturation, the $V_{1}^{\prime}$-lineshape is similar to that for the in-phase $V_{1}$-signal (see Fig. 1e), and becomes of the same polarity after phase-sensitive detection (cf. Eqs. [11], [16]). However, the dependences of the peak-to-peak amplitudes and peak-to-peak linewidths on the microwave $H_{1}$-field intensity are significantly different for the in-phase and out-of-phase components, in the latter higher region of $H_{1}$. As is seen from Figs. $2 \mathrm{~A}$ and $2 \mathrm{~B}$, the amplitude of the 

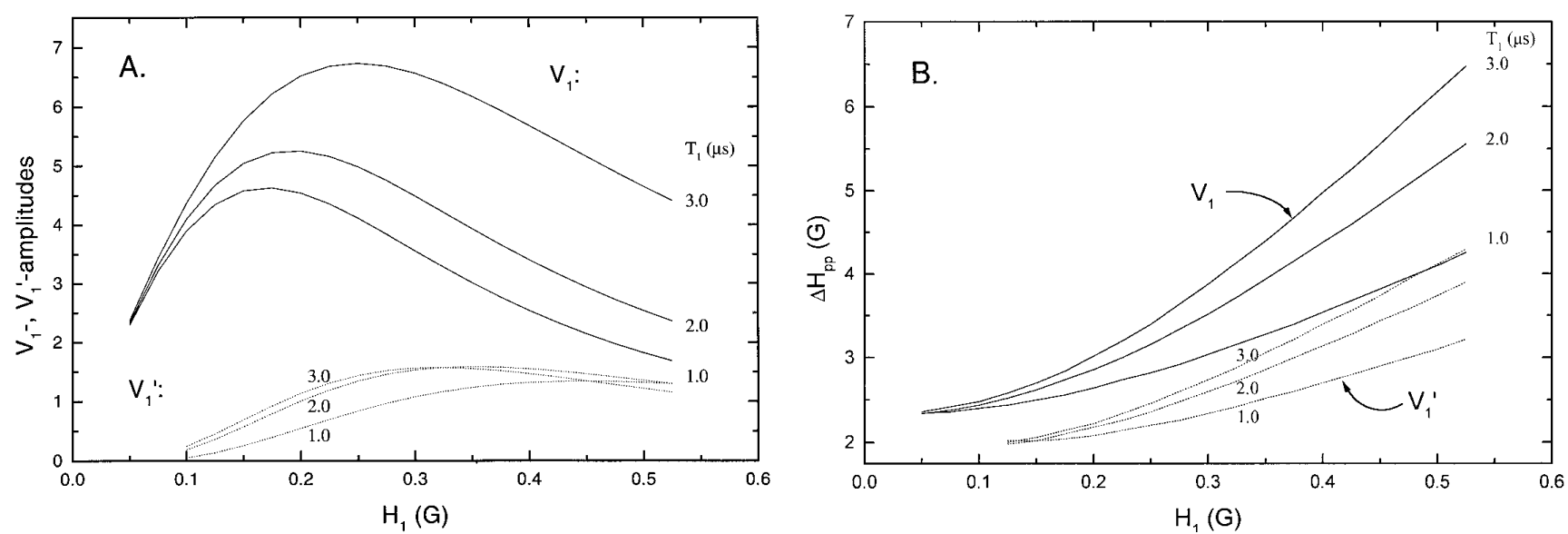

FIG. 2. Dependence of (A) the peak-to-peak amplitudes and (B) the peak-to-peak linewidths of the in-phase (full lines) and out-of-phase (dotted lines) first-harmonic absorption EPR spectra on the $H_{1}$-field strength. Spectra were simulated without approximation according to Eqs. [1] and [2] for the in-phase and out-of-phase spectra, respectively. The modulation frequency is $\omega_{m} / 2 \pi=10^{5} \mathrm{~s}^{-1}$ and the intrinsic homogeneous linewidth is $\Delta H\left(=1 / \gamma_{e} T_{2}\right)=2.0 \mathrm{G}$.

out-of-phase $V_{1}^{\prime}$-signal saturates at higher values of $H_{1}$, and the peak-to-peak linewidth increases less strongly with increasing $H_{1}$, than the in-phase $V_{1}$-amplitudes and linewidths. The out-of-phase signal in this region of $H_{1}$-values is considerably sharper than the in-phase signal, which contributes favorably to its relative amplitude.

The transition between the two regions, for which the outof-phase $V_{1}^{\prime}$ EPR spectrum has very different lineshapes, is determined by the condition $A+2 B=0$, and occurs at a microwave field specified by $h^{*^{2}}=\left(3+\omega^{\prime 2}\right)\left(a^{2}+\omega^{\prime 2}\right) /\left(\omega^{\prime 2}\right.$ $+2-a$ ) (see Eqs. [2], [3] and Ref. (15)). For the case $T_{2} \ll$ $T_{1}$, which usually holds for nitroxide spin labels, the transition takes place over a rather narrow interval of $H_{1}$-values (see Figs. 1a-1d), and the transition value of the microwave field $H_{1}^{*}$ can then be used for direct evaluation of $T_{1}$ :

$$
\gamma_{e}^{2} H_{1}^{* 2} \approx 1.5\left(1 / T_{1}^{2}+\omega_{m}^{2}\right)
$$

This requires precise determination of the $H_{1}$-field intensity at low microwave powers, and precise definition of the critical transition point. For shorter spin-lattice relaxation times $\left(T_{1} \leq\right.$ $0.5 \mu \mathrm{s})$, the transition interval broadens, and in this case the $T_{1}$-values can best be determined from lineshape simulations for $H_{1}$-values in the transition region.

\section{Experimental Dependence of $V_{1}^{\prime}$-Lineshape on $H_{1}$}

Both types of $V_{1}^{\prime}$-lineshapes described above can be realized experimentally. The out-of-phase first-harmonic EPR spectra of TEMPONE in a $90 \%$ glycerol/water mixture at $20^{\circ} \mathrm{C}$, measured at two different $H_{1}$-fields with a Zeeman modulation frequency of $100 \mathrm{kHz}$, are given in Figs. If and 1g. The microwave field corresponding to the transition from spectrum 1 a to spectrum $1 \mathrm{e}$ in this experimental system is $H_{1}^{*} \approx 0.054 \mathrm{G}$ (cf. Figs. $1 \mathrm{c}$ and 1f). The correspond- ing value of the effective spin-lattice relaxation time estimated from Eq. [17] is $T_{1} \approx 2.2 \mu \mathrm{s}$. It should be noted that the experimentally derived spin-lattice relaxation times are effective values, smaller than the intrinsic $T_{1}$ as determined by time-domain methods, because they contain contributions from nuclear relaxation (see, e.g., Ref. (18)). This point is treated in more detail elsewhere (14).

For the regime of shorter $T_{1}$-values, lineshape simulations were made in the transition region of $H_{1}$-values for 0.7 mol\% spin-labeled 16-PCSL phosphatidylcholine in fluid DMPC bilayer membranes. Experimental and simulated lineshapes are given as a function of $H_{1}$ in Fig. 3. The change in lineshape takes place over an interval of $H_{1}$ values broader than that obtained for the simulations with longer $T_{1}$ in Fig. 1. Effective values for the spin-lattice relaxation time of 16-PCSL that are established in fluid membranes from comparison with the simulated dependence of the $V_{1}^{\prime}$-lineshape on $H_{1}$ are given in part (a) of Table 1. It is interesting to note that the transition in lineshape of 16-PCSL occurs consistently at somewhat higher values of $H_{1}$ for the central $\left(M_{I}=0\right){ }^{14} \mathrm{~N}$ hyperfine manifold than for the low-field $\left(M_{I}=+1\right)$ manifold (see Fig. 3). This small but detectable difference, which gives rise to different effective values of $T_{1}$ for the different hyperfine components (see Table 1a), is most probably due to ${ }^{14} \mathrm{~N}$ nuclear relaxation $\left(\Delta M_{I}= \pm 1\right)$ which contributes to a greater extent to the central $M_{I}=0{ }^{14} \mathrm{~N}$ hyperfine component (e.g., Ref. (18)). Also for this reason, the effective values obtained for $T_{1}$ are lower than the intrinsic values of $T_{1}$ determined in comparable systems by saturation recovery EPR (14). It will be noted that the accuracy of determining effective $T_{1}$ values by this method depends essentially only on the accuracy of the determination of the absolute $H_{1}$-values, but requires precise setting of the modulation phase. 

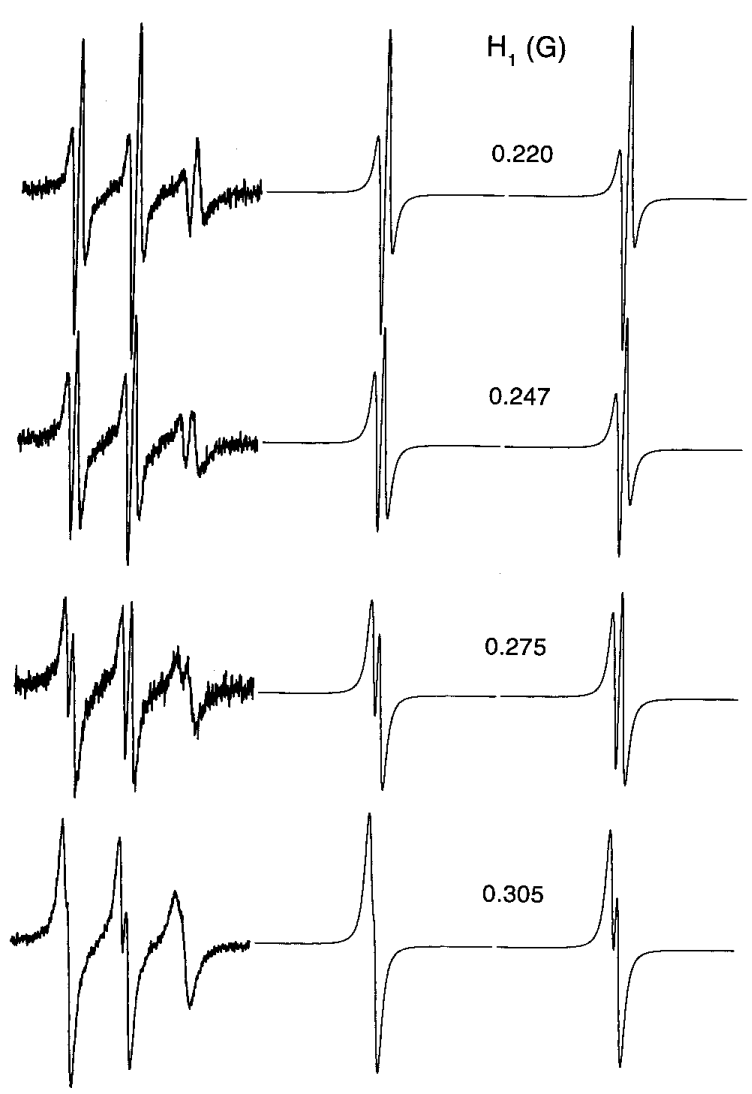

FIG. 3. Comparison of the $H_{1}$-dependence of experimental $V_{1}^{\prime}$-lineshapes with simulation. Left: 16-PCSL lipid spin label in DMPC membranes at $50^{\circ} \mathrm{C}$. Center and right: Exact simulations corresponding to the low-field and central hyperfine manifolds, with $T_{1}=0.23$ and $0.215 \mu \mathrm{s}$, respectively. Field scan width displayed is $60 \mathrm{G}$, in each case.

\section{Dependence of the First-Harmonic Out-of-Phase Intensities} on $\mathrm{H}_{1}$

The saturation of the intensity of the double-integrated in-phase absorption component specified by Eq. [9] is less than that for slow-passage conditions (i.e., $A<1+s$ ) because the $A$-term takes into account the rapid passage through the resonance at high Zeeman modulation frequencies (cf. Eq. [7]). In the limit of low modulation frequencies, i.e., $\omega^{\prime 2} \ll a$, then $A=1+s$ and the usual slow-passage expression for the double integral of the in-phase first-harmonic absorption is obtained. For low microwave saturation, when $B \approx-2$, $A \approx 1$, and $s \approx 0$, it follows from Eq. [11] that the out-of-phase/inphase integral intensity ratio is very small, i.e., $\rho_{1}^{\prime} \approx 0$.

The opposite situation, in which the $V_{1}^{\prime}$-lineshape is similar to that for the in-phase $V_{1}$-signal (see Fig. 1e), is realized already for rather moderate saturation, for instance, for $H_{1} \geq 0.2 \mathrm{G}$ with $T_{1} \geq$ $0.5 \mu \mathrm{s}$. In this case, under conditions for which $B \approx h^{2} /\left(a^{2}+\omega^{\prime 2}\right)$ and the approximation given by Eq. [7] for the $A$-term holds, the integral intensity ratio is given from Eq. [11] by

$$
\begin{aligned}
\rho_{1}^{\prime} & =-\frac{\omega_{m} T_{1}}{2} \\
& \times\left(\begin{array}{c}
T_{2} / T_{1}+\frac{s}{1+s+\omega_{m}^{2} T_{1}^{2}} \\
+\sqrt{(1+s)\left(1+\omega_{m}^{2} T_{1}^{2}\right)\left(1+s+\omega_{m}^{2} T_{1}^{2}\right)}
\end{array}\right) .
\end{aligned}
$$

For low modulation frequencies, corresponding to the condition $\omega_{m}^{2} T_{1}^{2} \ll 1$, further simplification is possible and the integral intensity ratio is then given by

$$
\rho_{1}^{\prime} \approx-\frac{\omega_{m} T_{1}}{2}\left(T_{2} / T_{1}+\frac{\gamma_{e}^{2} H_{1}^{2} T_{1} T_{2}}{2\left(1+\gamma_{e}^{2} H_{1}^{2} T_{1} T_{2}\right)}\right),
$$

where it is expected that the first term in Eqs. [18] and [19] is small, because $T_{2} / T_{1} \ll 1$.

The dependence on $H_{1}$-field strength of the normalized intensity and amplitude ratios of the out-of-phase first harmonic signal are given for different values of $T_{1}$ in Fig. 4. Exact numerical

TABLE 1

Effective Spin-Lattice Relaxation Times of the 16-PCSL Lipid Spin Label in DMPC Fluid-Phase Membranes at $30^{\circ}$ and $50^{\circ} \mathrm{C}$ Deduced from (a) Lineshape Simulations of the $V_{1}^{\prime}$-Spectra at $H_{1}$-field Strengths in the Transition Region, (b) Ratios of the

\begin{tabular}{|c|c|c|c|c|c|c|}
\hline $\begin{array}{c}T \\
\left({ }^{\circ} \mathrm{C}\right)\end{array}$ & \multicolumn{2}{|c|}{$\begin{array}{c}T_{1} \\
(\mu \mathrm{s})^{a}\end{array}$} & $\begin{array}{l}H_{1} \\
(\mathrm{G})\end{array}$ & $\rho_{1}^{\prime}$ & $\begin{array}{c}T_{1} \\
(\mu \mathrm{s})^{b}\end{array}$ & $\begin{array}{c}T_{1} \\
(\mu \mathrm{s})^{c}\end{array}$ \\
\hline & $\left(M_{I}=+1\right)$ & $\left(M_{I}=0\right)$ & & & & \\
\hline 30 & 0.25 & 0.23 & 0.615 & $2.51 \times 10^{-2}$ & 0.24 & $0.23(5)$ \\
\hline \multirow[t]{3}{*}{50} & 0.23 & $0.21(5)$ & 0.63 & $2.36 \times 10^{-2}$ & 0.22 & 0.23 \\
\hline & & & 0.59 & $2.09 \times 10^{-2}$ & 0.21 & \\
\hline & & & 0.50 & $1.62 \times 10^{-2}$ & 0.20 & \\
\hline
\end{tabular}
D ouble-Integrated Intensities of the Out-of-Phase $V_{1}^{\prime}$-Spectra to the In-Phase $V_{1}$-Spectra, and (c) Saturation Parameters of the In-Phase $V_{1}$ EPR Absorption, $\omega_{m} / 2 \pi=10^{5} \mathrm{~s}^{-1}$

\footnotetext{
${ }^{a}$ Determined from lineshape simulations without approximation of the $M_{I}=+1$ and $M_{I}=0$ manifolds of the $V_{1}^{\prime}$-spectra, in the range $0.21 \mathrm{G}<H_{1}<0.31 \mathrm{G}$.

${ }^{b}$ Determined from the double-integrated intensity ratio, $\rho_{1}^{\prime}$, using simulations without approximation.

${ }^{c}$ Determined by fitting the power saturation curves of the double-integrated $V_{1}$-intensities as a function of $H_{1}$, and fitting the $V_{1}$-lineshapes to obtain the
} Lorentzian linewidth (cf. Ref. (13)). 

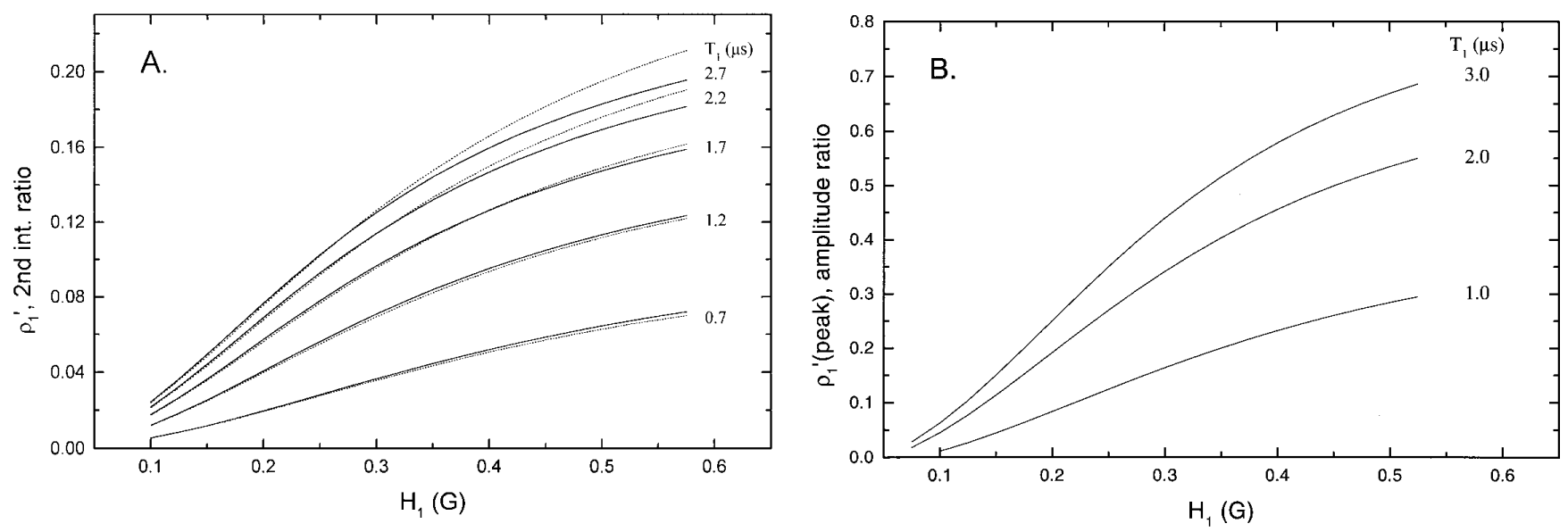

FIG. 4. Dependence of the out-of-phase to in-phase ratios of (A) the double-integrated spectral intensities and (B) the spectral peak amplitudes of the first-harmonic absorption EPR spectra on the $H_{1}$-field strength. Spectra were simulated without approximation according to Eqs. [1] and [2] for the values of $T_{1}$ indicated and their intensities evaluated (full lines). The integral intensities calculated from the approximate analytical Eq. [18] are given by the dotted lines in (A). The modulation frequency is $\omega_{m} / 2 \pi=10^{5} \mathrm{~s}^{-1}$ and the intrinsic homogeneous linewidth is $\Delta H\left(=1 / \gamma_{e} T_{2}\right)=2.0 \mathrm{G}$.

results obtained directly from Eqs. [1] and [2] are compared with calculations based on the approximate analytical expression given by Eq. [18]. The analytical expression is seen to agree well with the exact solution for $\omega_{m} T_{1}<1$ and to deviate from this for $\omega_{m} T_{1}$ $>1$, in line with the approximations that were made in deriving the former. The ratio of the out-of-phase to in-phase integral intensities increases strongly with increasing $H_{1}$ and tends to level off at high values of $H_{1}$ (i.e., for $\left.s \gg 1\right)$. High values of $H_{1}\left(H_{1} \geq\right.$ $0.5 \mathrm{G})$ are needed in order to obtain appreciable $V_{1}^{\prime}$-signal strengths and, correspondingly, values of the intensity ratio with good signal-to-noise ratio.

The dependence of the peak amplitude ratio on $H_{1}$ is qualitatively similar to that for the integrated intensity ratio. The amplitude ratio increases strongly with increasing $H_{1}$, in agreement with Eq. [12], such that comparable values of the out-ofphase and in-phase amplitude can be achieved at high $H_{1}$ for long $T_{1}$-values.

\section{Dependence of the First-Harmonic Out-of-Phase Intensities on $T_{1}$ (and $T_{2}$ )}

It follows from Eqs. [18] and [19] that the ratio of the integrated intensities, $\rho_{1}^{\prime}$, increases with increasing spin-lattice relaxation time as $T_{1}^{2}$ at moderate degrees of saturation, and is linear in $T_{1}$ at higher degrees of saturation. Thus, the out-ofphase non-linear, first-harmonic spectrum is more sensitive for $T_{1}$-measurements than are conventional progressive saturation experiments on the first-harmonic in-phase spectrum. The double-integrated intensity of the latter is determined by the factor $\sqrt{A}+\sqrt{(1+s)}$, which depends on spin-lattice relaxation time approximately as $T_{1}^{1 / 2}$ (see Eq. [9]).

The predicted dependences on spin-lattice relaxation time of the normalized double-integrated intensity of the out-of-phase first-harmonic absorption spectrum are given in Fig. 5, for Zeeman modulation frequencies of 100 and $25 \mathrm{kHz}$. Corre- sponding dependences on $T_{1}$ for the normalized peak amplitudes of the out-of-phase first-harmonic absorption spectrum are given in Fig. 6. Again, the dependences predicted from the approximate analytical solution (Eq. [18]) agree with the exact numerical solution (cf. Eqs. [1] and [2]) for $\omega_{m} T_{1}<1$, but deviate considerably for $\omega_{m} T_{1}>1$. (In particular, the approximate expression does not exhibit such pronounced leveling off of the intensity ratio at high $T_{1}$ ). The optimum $T_{1}$-sensitivity at a modulation frequency of $100 \mathrm{kHz}$ is in the range of $T_{1}=$ 0.5-3 $\mu$ s (Fig. 5A). This interval, however, can be increased considerably to higher values of $T_{1}$ by using lower modulation frequencies. In fact, as seen from Eq. [19], for $\omega_{m}^{2} T_{1}^{2} \ll 1$ the dependence of the integrated intensity ratio no longer levels off with increasing $T_{1}$. For a Zeeman modulation frequency of 25 $\mathrm{kHz}$, the integral intensity ratio increases approximately linearly with $T_{1}$ for values up to $T_{1}=5-6 \mu \mathrm{s}$ and continues to increase steadily beyond this (see Fig. 5B). Moreover, the intensity ratios at high microwave $H_{1}$-fields can become comparable to, or even greater than, those at a modulation frequency of $100 \mathrm{kHz}$. These conditions favor using high values of $H_{1}$ and lower modulation frequencies for $T_{1}$-determinations from non-linear first-harmonic EPR spectra.

It is found from Eq. [18] and the form of Fig. 5 that the dependence of the integral intensity ratio on the $T_{1}$-relaxation time can be approximated by the semi-empirical expression

$$
\rho_{1}^{\prime}=\rho_{1}^{o^{\prime}}+\frac{a_{1}^{\prime} T_{1}^{m}}{1+b_{1}^{\prime} T_{1}^{m}},
$$

where the parameters $a_{1}^{\prime}$ and $b_{1}^{\prime}$ depend on $H_{1}$, the $T_{2}$-relaxation time, and the Zeeman modulation frequency. The functional form that is given in Eq. [20] can be used to parameterize the calibration of the measured integral intensity ratios in terms of 

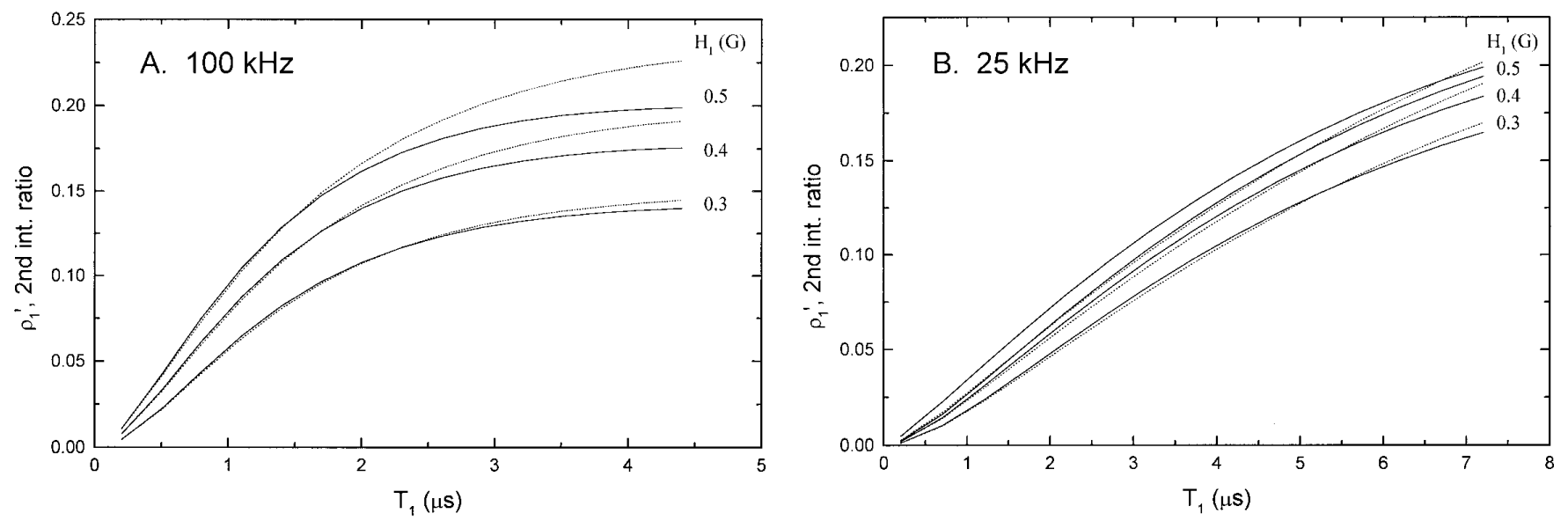

FIG. 5. Dependence on spin-lattice relaxation time, $T_{1}$, of the out-of-phase to in-phase ratio, $\rho_{1}^{\prime}$, of the double-integrated intensity for the first-harmonic absorption EPR spectrum. (A) Modulation frequency, $\omega_{m} / 2 \pi=10^{5} \mathrm{~s}^{-1}$. (B) Modulation frequency, $\omega_{m} / 2 \pi=2.5 \times 10^{4} \mathrm{~s}^{-1}$; for the values of the $H_{1}$-field shown Full lines are exact calculations from Eqs. [1] and [2] and dotted lines are from the approximation given by Eq. [18]. The intrinsic linewidth is $\Delta H$ (=1/ $\left.\gamma_{e} T_{2}\right)$ $=2.0 \mathrm{G}$, except for the two full lines corresponding to $H_{1}=0.5 \mathrm{G}$ given in (B), which represent the limits for $\Delta H=1.0 \mathrm{G}$ (upper) and $\Delta H=2.5 \mathrm{G}$ (lower).

the $T_{1}$-relaxation time, where $a_{1}^{\prime}, b_{1}^{\prime}$, and the exponent $m$ are the calibration parameters. These values are given in Table 2 , along with corresponding values for an equivalent calibration of the amplitude ratios from Fig. 6 . In all cases, the $T_{1^{-}}$ dependences in Figs. 5 and 6 obtained from exact simulations are well fit by the empirical form given by Eq. [20], with the parameters given in Table 2. Differences of the fits from the full lines shown would be barely discernible in these figures.

From comparison of Fig. 6 with Fig. 5 it is seen that the dependence of the first-harmonic out-of-phase/in-phase amplitude ratio, $\rho_{1}^{\prime}$ (peak) on $T_{1}$ is qualitatively similar to that of the ratio of the integrated intensities, $\rho_{1}^{\prime}$. Again, the amplitude ratio measured at fixed field position also increases as $T_{1}^{2}$ and as $T_{1}$ at moderate and higher saturation, respectively, as for the integral ratio (see
Eq. [13]). Moreover, the sensitivity of the amplitude ratio to $T_{1}$ is somewhat better than that for $\rho_{1}^{\prime}$, at higher values of $T_{1}$. As for the integrated intensity ratio, the sensitivity of the amplitude ratio to longer values of $T_{1}$ increases at lower Zeeman modulation frequencies (see Figs. 6A, and 6B). The out-of-phase first-harmonic amplitudes are seen to be comparable to the in-phase amplitudes at high values of $H_{1}$ and of $T_{1}$ (i.e., $\rho_{1}^{\prime}($ peak) $\sim 1$ ). This ensures good signal-to-noise ratio in the non-linear amplitude measurements, under these conditions. As mentioned above, the calibration parameters for the $T_{1}$-dependence of the amplitude parameters are given in Table 2.

For the non-linear first-harmonic EPR method to be particularly suited to measurements of spin-lattice relaxation, it is desirable that the dependence of the out-of-phase/in-phase integral and
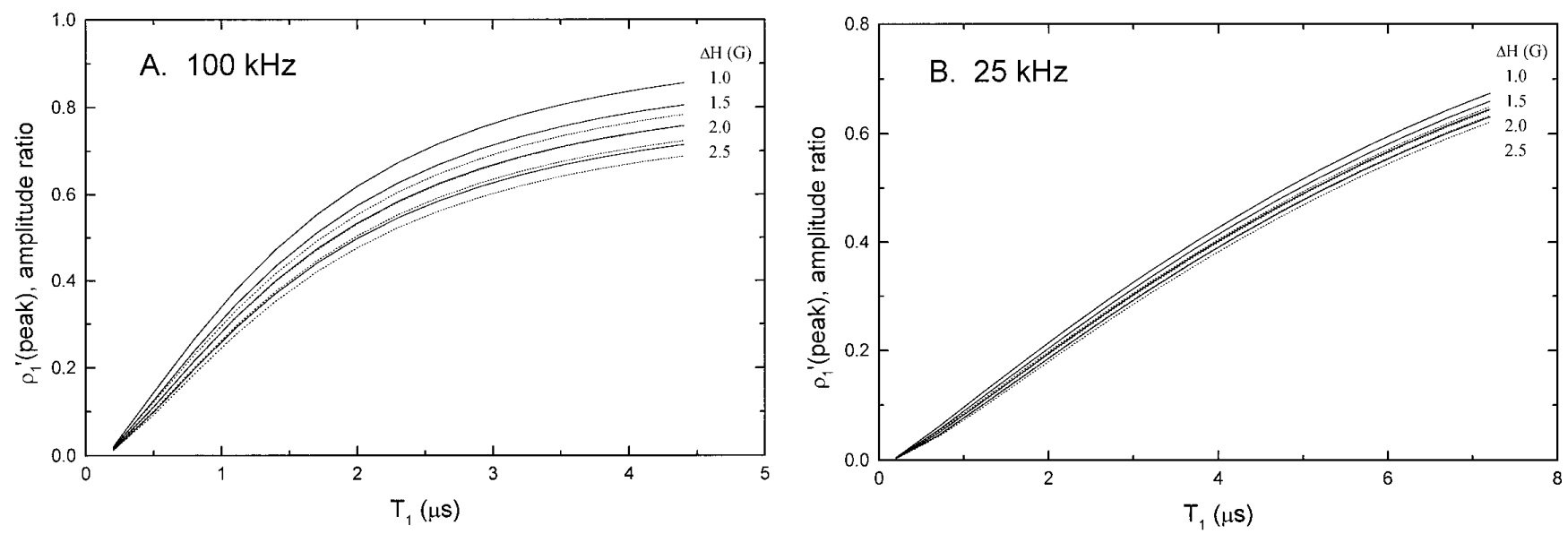

FIG. 6. Dependence of the out-of-phase to in-phase ratio, $\rho_{1}^{\prime}$ (peak), of the peak amplitudes for the first-harmonic absorption EPR spectrum on spin-lattice relaxation time, $T_{1}$, at a microwave field of $H_{1}=0.5 \mathrm{G}$. (A) Modulation frequency, $\omega_{m} / 2 \pi=10^{5} \mathrm{~s}^{-1}$. (B) Modulation frequency, $\omega_{m} / 2 \pi=2.5 \times 10^{4} \mathrm{~s}-1$. Full lines are from simulations without approximation using Eqs. [1] and [2] with the intrinsic homogeneous linewidths $\Delta H\left(=1 / \gamma_{e} T_{2}\right)$ shown, and dotted lines are with an additional Gaussian inhomogeneous broadening of $1.4 \mathrm{G}$. 
TABLE 2

Parameterization of $\mathrm{C}$ alibrations for the $T_{1}-\mathrm{D}$ ependence of the First-H armonic A bsorption Out-of-Phase/In-Phase R atio in Terms of Eq. [20] for Both the D ouble Integral Intensities and the Amplitudes $^{a}$

\begin{tabular}{lcccccc}
\hline $\begin{array}{c}\omega_{m} / 2 \pi \\
(\mathrm{kHz})\end{array}$ & $\begin{array}{c}H_{1} \\
(\mathrm{G})\end{array}$ & $\begin{array}{c}\Delta H \\
(\mathrm{G})\end{array}$ & $\rho_{1}^{\mathrm{o}^{\prime}}$ & $a_{1}^{\prime}$ & $b_{1}^{\prime}$ & $m$ \\
\hline Integrals & & & & & & \\
100 & 0.5 & 2.0 & 0.0067 & 0.1562 & 0.754 & 2.0 \\
100 & 0.4 & 2.0 & 0.0041 & 0.1265 & 0.680 & 2.0 \\
100 & 0.3 & 2.0 & 0.0019 & 0.0894 & 0.590 & 2.0 \\
25 & 0.5 & 2.0 & 0.0026 & 0.0347 & 0.0967 & 1.3 \\
25 & 0.4 & 2.0 & 0.0035 & 0.0315 & 0.0897 & 1.3 \\
25 & 0.3 & 2.0 & 0.0045 & 0.0263 & 0.0760 & 1.3 \\
& & & & & & \\
Amplitudes & & & & & & \\
100 & 0.5 & 1.0 & -0.0160 & 0.553 & 0.538 & 1.6 \\
100 & 0.5 & 1.5 & -0.0170 & 0.496 & 0.507 & 1.6 \\
100 & 0.5 & 2.0 & -0.0177 & 0.489 & 0.483 & 1.6 \\
100 & 0.5 & 2.5 & -0.0179 & 0.410 & 0.464 & 1.6 \\
25 & 0.5 & 1.0 & -0.0037 & 0.1055 & 0.0787 & 1.3 \\
25 & 0.5 & 1.5 & -0.0064 & 0.1014 & 0.0753 & 1.3 \\
25 & 0.5 & 2.0 & -0.0086 & 0.0975 & 0.0720 & 1.3 \\
25 & 0.5 & 2.5 & -0.0100 & 0.0938 & 0.0690 & 1.3 \\
\hline
\end{tabular}

Note. In the case of the amplitude ratios, a form identical to Eq. [20] is assumed. Calibrations are obtained from fitting the results of exact simulations given in Figs. 5 and 6 for integrals and amplitude ratios, respectively.

${ }^{a}$ Parameters $a_{1}^{\prime}$ and $b_{1}^{\prime}$ are given for $T_{1}$ in $\mu$ s.

amplitude ratios on the transverse relaxation time, $T_{2}$, be relatively slight. It is seen from Eqs. [18] and [19] that the main contribution to the integral ratio, for $T_{2} / T_{1} \ll 1$, comes from the second term in the outer brackets. The dependence of this term on $T_{2}$ becomes weaker at lower modulation frequencies and at increasing extents of microwave saturation. In fact, for $\omega_{m}^{2} T_{1}^{2} \ll 1$, when Eq. [19] holds, the integral ratio, $\rho_{1}^{\prime}$, must be virtually independent of $T_{2}$ at large values of the saturation parameter, $s=$ $\gamma_{e}^{2} H_{1}^{2} T_{1} T_{2}$. This conclusion is confirmed quantitatively by exact numerical calculations performed for $\omega_{m} / 2 \pi=25 \mathrm{kHz}$ (see Fig. 5B for $H_{1}=0.5 \mathrm{G}$ ). As for the integral ratio, the amplitude ratio $\rho_{1}^{\prime}$ (peak) is also relatively insensitive to the homogeneous broadening $\left(\Delta H=1 / \gamma_{e} T_{2}\right)$, and this residual sensitivity diminishes yet further at lower Zeeman modulation frequencies (see Figs. 6A and 6B). The sensitivity of non-linear first harmonic EPR to $T_{2}$ is therefore considerably less than that of the conventional saturation parameter $\left(\gamma_{e}^{2} H_{1}^{2} T_{1} T_{2}\right)$ in progressive saturation measurements, and than that of the standard second-harmonic non-linear ST EPR (cf. Ref. (11)).

The effect of Gaussian inhomogeneous broadening on the first-harmonic out-of-phase/in-phase amplitude ratio is also given in Fig. 6. For Gaussian peak-to-peak linewidths of $\Delta H_{p p}^{G}$ $=1.4 \mathrm{G}$, which is the maximum value for different nitroxide spin labels in the rapid motion region $(19,20)$, the dependence of the amplitude ratio on $T_{1}$ is close to that for the case of homogeneous broadening. This is possibly due to a partial cancellation of the effects of inhomogeneous broadening on the out-of-phase and in-phase signals when taking the amplitude ratio, $\rho_{1}^{\prime}$ (peak). The out-of-phase/in-phase integrated intensity ratio, $\rho_{1}^{\prime}$, is completely insensitive to inhomogeneous broadening, just as in progressive saturation measurements (13).

\section{Experimental Determination of Out-of-Phase/In-Phase Ratios for First-Harmonic Absorption Amplitudes and Integrated Intensities}

Determinations of the non-linear first-harmonic EPR spectra have been made for a small nitroxide spin label (TEMPONE) in homogeneous solution, and for spin-labeled lipids (5- and 16-PCSL) in phospholipid bilayer membranes. Both the dependence of the saturation behavior on the microwave $H_{1}$-field and the spin-lattice relaxation enhancement by paramagnetic ions have been investigated.

The dependence of the out-of-phase/in-phase ratios on $H_{1}$-field strength for TEMPONE in a $90 \%$ glycerol-water solution at $20^{\circ} \mathrm{C}$ is given in Figs. 7A and 7B, for the first-harmonic absorption integrated intensities and peak-to-peak amplitudes, respectively. Experimental $V_{1}^{\prime}$-spectra for this system at a modulation frequency of $100 \mathrm{kHz}$ and lower values of $H_{1}$ were shown in Figs. 1f and $1 \mathrm{~g}$. The Zeeman modulation frequency used for obtaining the data given in Fig. 7 is $25 \mathrm{kHz}$, which is appropriate to determining longer spin-lattice relaxation times from the out-of-phase intensities (as opposed to lineshapes). The inhomogeneous broadening is known to be very small for the TEMPONE spin label: $\Delta H_{p p}^{G}=$ $0.215 \mathrm{G}$ (19). The Lorentzian spin packet width, $\Delta H\left(=1 / \gamma_{e} T_{2}\right)=$ $0.9 \mathrm{G}$, was obtained from spectral simulations of the in-phase $V_{1}$ EPR spectra at subsaturating microwave power. This value, together with Eqs. [1] and [2], was used to calculate the predicted dependences of the integral and amplitude ratios on $H_{1}$-field strength. For the intensities, a sum was made over all three hyperfine lines and, for the amplitudes, the low-field line was used. A satisfactory fit to the experimental dependences is obtained for effective values of $T_{1}=3.0 \mu \mathrm{s}$ (Fig. 7A) and $T_{1}=2.85$ $\mu \mathrm{s}$ (Fig. 7B). These effective values of $T_{1}$ obtained from the integral and amplitude ratios are close to one another and are in reasonably satisfactory agreement with the estimate of $\approx 2.2 \mu \mathrm{s}$ obtained from the $V_{1}^{\prime}$-lineshape transition (see Eq. [17] above, and Figs. 1c,f).

The 16-PCSL spin label in fluid lipid bilayer membranes also gives rise to sharp spectra in which the three hyperfine components are non-overlapping and devoid of anisotropy (see Fig. 3). The out-of-phase $V_{1}^{\prime}$-intensities at high $H_{1}$-fields have been used to determine the effective $T_{1}$-relaxation times, for 16-PCSL in this membrane system, as described previously for TEMPONE in homogeneous solution. In this case, a Zeeman modulation frequency of $100 \mathrm{kHz}$, which is appropriate for shorter $T_{1}$-values, was used. The results of these determinations are given in Table 1 above. It is seen that the effective $T_{1}$-relaxation times obtained by the out-of-phase intensity method at high $H_{1}$ agree rather well with those derived from 

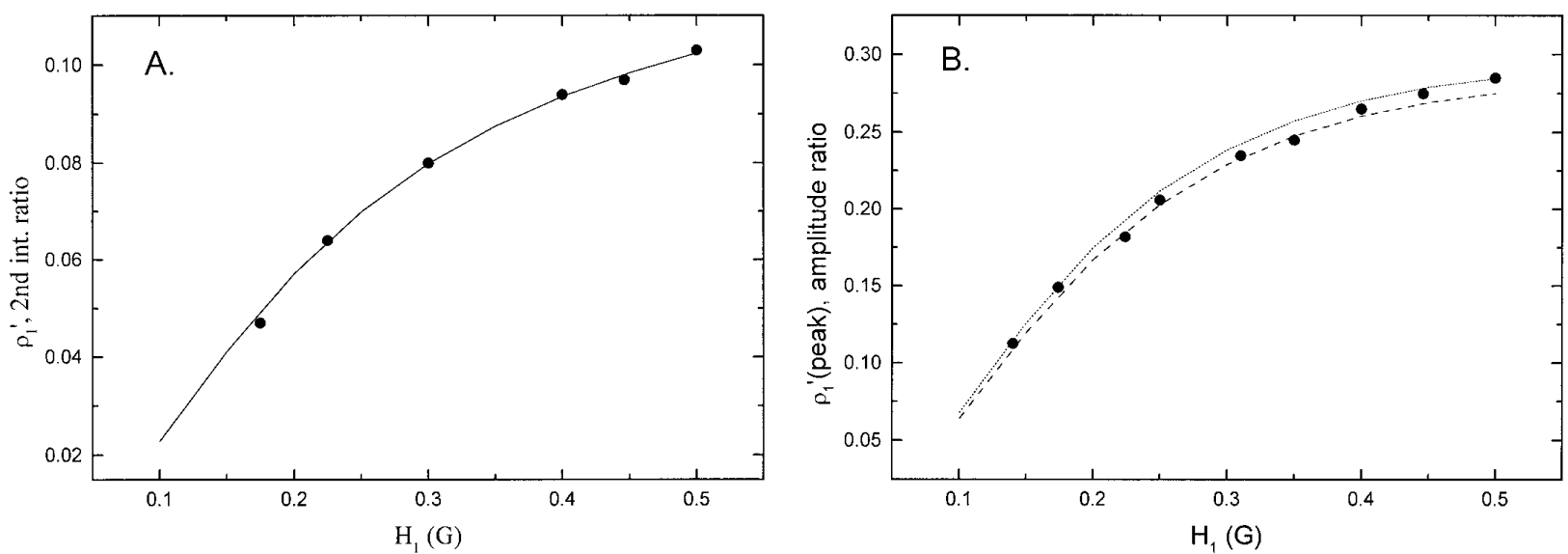

FIG. 7. Experimental dependence on $H_{1}$-field strength of the out-of-phase to in-phase ratios of (A) the double-integrated intensities and (B) the peak-to-peak low-field amplitudes in the first-harmonic absorption EPR spectra from the TEMPONE spin label in $90 \%$ glycerol/water solution at $20^{\circ} \mathrm{C}$. The modulation frequency is $\omega_{m} / 2 \pi=2.5 \times 10^{4} \mathrm{~s}^{-1}$. The lines give the predicted dependence without approximation according to Eqs. [1] and [2] with a homogeneous linewidth of $\Delta H=0.9 \mathrm{G}$ and (A) $T_{1}=3.0 \mu \mathrm{s}$ (full line); (B) $T_{1}=2.9 \mu \mathrm{s}$ (dotted line) or $T_{1}=2.8 \mu \mathrm{s}$ (dashed line).

lineshape simulations at lower $H_{1}$-fields. They are also in agreement with the values obtained from conventional progressive saturation of the double-integrated intensity of the inphase $V_{1}$-spectra, combined with linewidth measurements, that are given in the same table. The effective spin-lattice relaxation times are all rather short, in comparison with timedomain measurements on comparable systems, most probably because of contributions from Heisenberg spin exchange (20) and possibly also nuclear relaxation (18).

The effective rotational correlation time for the 5-PCSL phosphatidylcholine spin label in gel-phase DPPC bilayer membranes at $15^{\circ} \mathrm{C}$, as estimated from the ST EPR spectral lineshapes, is in the region of $\geq 10 \mu$ s (e.g., Ref. (21)). This value corresponds to the rigid limit for the first-harmonic spectral lineshapes, i.e., to the no-motion case. Analysis of the low-field spectral component with a Gaussian-Lorentzian deconvolution showed that the lineshape is essentially Lorentzian, with a homogeneous width of $\Delta H\left(=1 / \gamma_{e} T_{2}\right) \approx 3.0 \mathrm{G}$. This width was virtually unchanged in the presence of $\mathrm{Ni}^{2+}$ ions. The out-of-phase/in-phase ratios of the $V_{1}$-EPR spectra from samples containing increasing concentrations of $\mathrm{Ni}^{2+}$ are given in Table 3, for the amplitudes of the low-field maxima, $\rho_{1}^{\prime}\left(M_{I}=+1\right)$, and for the double-integrated intensities of the entire spectra. The first-harmonic absorption EPR spectra $\left(V_{1}^{\prime}\right.$ and $V_{1}$ ) were recorded with a Zeeman modulation frequency of $100 \mathrm{kHz}$, and high $H_{1}$-field strengths of 0.5 and $0.4 \mathrm{G}$. Values for the effective $T_{1}$-relaxation time derived from the amplitude and integrated intensity ratios of the non-linear spectra are compared with those obtained from progressive power saturation experiments in Table 3. For the latter, the effective $T_{1}$ was calculated from the saturation factor and the $T_{2}$-value obtained from the homogeneous linewidth. The effective values of $T_{1}$ obtained by the different methods are in reasonable agreement, and consistent values are obtained from the non-linear first- harmonic spectra at different $H_{1}$-field strengths. The effective spin-lattice relaxation rate, $1 / T_{1}$, obtained from the out-ofphase/in-phase ratios increases linearly with $\mathrm{Ni}^{2+}$ ion concentration, as expected for a true paramagnetic relaxation enhancement (see Fig. 8). The non-linear first-harmonic EPR spectra are therefore seen to be suitable for determining spin-label $T_{1}$-relaxation enhancements in biological systems.

TABLE 3

Effective Spin-Lattice Relaxation Times of the 5-PCSL Lipid Spin Label in D PPC Gel-Phase Membranes at $15^{\circ} \mathrm{C}$ Deduced from the Ratio (a) of the Low-Field Amplitudes and (b) the DoubleIntegrated Intensities of the Out-of-Phase $\left(V_{1}^{\prime}\right)$ and In-Phase $\left(V_{1^{-}}\right)$ Spectra, and Also from (c) the Saturation Parameters of the In-Phase $V_{1}$ E PR A bsorption, $\omega_{m} / 2 \pi=10^{5} \mathrm{~s}^{-1}$

\begin{tabular}{ccccccc}
\hline $\begin{array}{c}{\left[\mathrm{NiCl}_{2}\right]} \\
(\mathrm{m} M)\end{array}$ & $\begin{array}{c}H_{1} \\
(\mathrm{G})\end{array}$ & $\rho_{1}^{\prime}\left(M_{I}=+1\right)$ & $\begin{array}{c}T_{1} \\
(\mu \mathrm{s})^{a}\end{array}$ & $\rho_{1}^{\prime}$ & $\begin{array}{c}T_{1} \\
(\mu \mathrm{s})^{b}\end{array}$ & $\begin{array}{c}T_{1} \\
(\mu \mathrm{s})^{c}\end{array}$ \\
\hline 0 & 0.4 & 0.158 & 1.2 & 0.094 & 1.5 & 1.7 \\
& 0.5 & 0.168 & 1.3 & 0.11 & 1.4 & \\
5 & 0.4 & 0.088 & 0.8 & 0.062 & 1.0 & 1.1 \\
& 0.5 & 0.1285 & 0.86 & 0.08 & 1.0 & \\
& & & & & & \\
& & & & & & \\
& & & & & & \\
20 & 0.5 & 0.108 & 0.76 & 0.052 & 0.69 & 0.9 \\
& 0.4 & 0.065 & 0.64 & 0.024 & 0.5 & \\
30 & 0.5 & 0.0665 & 0.54 & 0.035 & 0.53 & \\
& 0.4 & 0.05 & 0.53 & 0.019 & 0.43 & 0.55 \\
& 0.5 & 0.06 & 0.5 & 0.0324 & 0.48 & \\
\hline
\end{tabular}

${ }^{a}$ Determined from the ratio $\rho_{1}^{\prime}\left(M_{I}=+1\right)$, of the low-field peak amplitudes.

${ }^{b}$ Determined from the double-integrated intensity ratio, $\rho_{1}^{\prime}$.

${ }^{c}$ Determined by fitting the power saturation curves of the double-integrated $V_{1}$-intensities as a function of $H_{1}$, and fitting the $V_{1}$-lineshapes to obtain the Lorentzian linewidth (cf. Ref. (13)). 


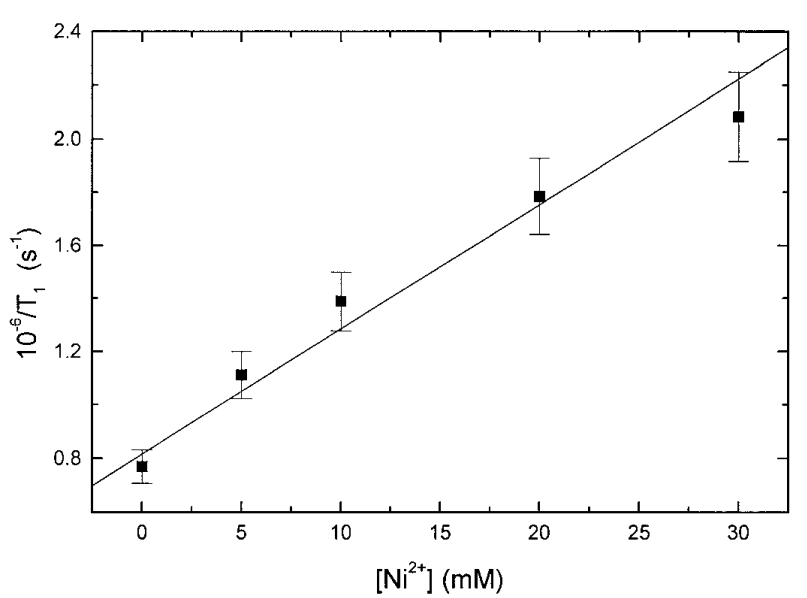

FIG. 8. Dependence of the effective spin-lattice relaxation rate, $1 / T_{1}$, deduced from the ratio of the integrated first-harmonic intensities, $\rho_{1}^{\prime}$, on aqueous $\mathrm{Ni}^{2+}$ ion concentration for the 5-PCSL phosphatidylcholine spin label in phospholipid bilayer membranes of DPPC at $15^{\circ} \mathrm{C}$. Modulation frequency: $\omega_{m} / 2 \pi=10^{5} \mathrm{~s}^{-1}$. The line is a linear regression to the data points.

\section{CONCLUSION}

Both spectral simulations and experimental results obtained on model systems show that the out-of-phase components of the first-harmonic absorption EPR spectra are particularly well suited for the determination of spin-lattice relaxation enhancements of spin labels. Special advantages of the non-linear first-harmonic EPR spectra are: (i) the relatively high signal intensity; (ii) the relative insensitivity to $T_{2}$-relaxation; and (iii) the ability to tune the range of $T_{1}$-relaxation rates to which the spectral intensities are sensitive by varying the Zeeman modulation frequency, while still maintaining adequate signal strengths. The method therefore should find useful application to spin-labeled biological systems.

\section{ACKNOWLE DGMENTS}

This work was supported partly by the Deutsche Forschungsgemeinschaft. The work of V. A. Livshits was supported in part by the Russian Foundation for Basic Research, under Grant 95-04-09604.

\section{REFERENCES}

1. A. Abragam, "The Principles of Nuclear Magnetism" (W. C. Marshall and D. H. Wilkinson, Eds.), Oxford Univ. Press, Oxford (1961).

2. D. Marsh, Exchange and dipolar spin-spin interactions and rotational diffusion in saturation transfer EPR spectroscopy, Appl. Magn. Reson. 3, 53- 65 (1992).

3. D. Marsh, Progressive saturation and saturation transfer ESR for measuring exchange processes of spin-labelled lipids and proteins in membranes, Chem. Soc. Rev. 22, 329-335 (1993).

4. W. L. Hubbell and C. Altenbach, Site-directed spin-labeling of membrane proteins, in “Membrane Protein Structure. Experimental
Approaches" (S. H. White, Ed.), pp. 224-248, Oxford Univ. Press, New York/Oxford (1994).

5. W. L. Hubbell and C. Altenbach, Investigation of structure and dynamics in membrane proteins using site-directed spin labeling, Curr. Opin. Struct. Biol. 4, 566-573 (1994).

6. T. Páli, R. Bartucci, L. I. Horváth, and D. Marsh, Distance measurements using paramagnetic ion-induced relaxation in the saturation transfer electron spin resonance of spin-labeled biomolecules. Application to phospholipid bilayers and interdigitated gel phases, Biophys. J . 61, 1595-1602 (1992).

7. M. M. E. Snel and D. Marsh, Membrane location of apocytochrome $\mathrm{c}$ and cytochrome $\mathrm{c}$ determined from lipid-protein spin exchange interactions by continuous wave saturation electron spin resonance, Biophys. J . 67, 737-745 (1994).

8. J. S. Hyde, W. Froncisz, and C. Mottley, Pulsed ELDOR measurement of nitrogen $T_{1}$ in spin labels, Chem. Phys. Lett. 110, 621- 625 (1984).

9. B. H. Robinson, D. A. Haas, and C. Mailer, Molecular dynamics in liquids: Spin-lattice relaxation of nitroxide spin labels, Science 263, $490-493$ (1994)

10. D. D. Thomas, L. R. Dalton, and J. S. Hyde, Rotational diffusion studied by passage saturation transfer electron paramagnetic resonance, J . Chem. Phys. 65, 3006-3024 (1976).

11. T. Páli, V. A. Livshits, and D. Marsh, Dependence of saturationtransfer EPR intensities on spin-lattice relaxation, J. Magn. Reson. B 113, 151-159 (1996).

12. D. Marsh and L. I. Horváth, Influence of Heisenberg spin exchange on conventional and phase-quadrature EPR lineshapes and intensities under saturation, J. Magn. Reson. 97, 13-26 (1992).

13. T. Páli, L. I. Horváth, and D. Marsh, Continuous-wave saturation of two-component, inhomogeneously broadened, anisotropic EPR spectra, J. Magn. Reson. A 101, 215-219 (1993).

14. V. A. Livshits, T. Páli and D. Marsh, Relaxation time determinations by progressive saturation EPR: Effects of molecular motion and Zeeman modulation for spin labels, J. Magn. Reson. 133, 79-91 (1998).

15. K. Halbach, Über eine neue Methode zur Messung von Relaxationszeiten und über den Spin von $\mathrm{Cr}^{53}$, Helv. Phys. Acta 27, 259-282 (1954).

16. D. Marsh and A. Watts, Spin-labeling and lipid-protein interactions in membranes, in "Lipid-Protein Interactions" (P. C. J ost, and O. H. Griffith, Eds.), pp. 53-126, Wiley-Interscience, New York (1982).

17. P. Fajer and D. Marsh, Microwave and modulation field inhomogeneities and the effect of cavity $Q$ in saturation transfer ESR spectra. Dependence on sample size, J . Magn. Reson. 49, 212224 (1982)

18. D. Marsh, Influence of nuclear relaxation on the measurement of exchange frequencies in CW saturation EPR studies. J. Magn. Reson. 99, 332-337 (1992).

19. B. L. Bales, Inhomogeneously broadened spin-label spectra, in "Biological Magnetic Resonance" (L. J. Berliner, and J. Reuben, Eds.), Vol. 8, Chap. 2, pp. 77-130, Plenum, New York (1989).

20. J -H. Sachse, M. D. King, and D. Marsh, ESR determination of lipid diffusion coefficients at low spin-label concentrations in biological membranes, using exchange broadening, exchange narrowing, and dipole-dipole interactions, J. Magn. Reson. 71, 385-404 (1987).

21. D. Marsh, Molecular motion in phospholipid bilayers in the gel phase: Long axis rotation, Biochemistry 19, 1632-1637 (1980). 